PROCEEDINGS OF THE

AMERICAN MATHEMATICAL SOCIETY

Volume 128, Number 1, Pages 141-147

S 0002-9939(99)04993-X

Article electronically published on June 30, 1999

\title{
INEQUALITIES FOR THE GAMMA FUNCTION
}

\author{
HORST ALZER
}

(Communicated by Hal L. Smith)

AbStract. We prove the following two theorems:

(i) Let $M_{r}(a, b)$ be the $r$ th power mean of $a$ and $b$. The inequality

$$
M_{r}(\Gamma(x), \Gamma(1 / x)) \geq 1
$$

holds for all $x \in(0, \infty)$ if and only if $r \geq 1 / C-\pi^{2} /\left(6 C^{2}\right)$, where $C$ denotes Euler's constant. This refines results established by W. Gautschi (1974) and the author (1997).

(ii) The inequalities

$$
x^{\alpha(x-1)-C}<\Gamma(x)<x^{\beta(x-1)-C}
$$

are valid for all $x \in(0,1)$ if and only if $\alpha \leq 1-C$ and $\beta \geq\left(\pi^{2} / 6-C\right) / 2$, while (*) holds for all $x \in(1, \infty)$ if and only if $\alpha \leq\left(\pi^{2} / 6-C\right) / 2$ and $\beta \geq 1$. These bounds for $\Gamma(x)$ improve those given by G. D. Anderson an S.-L. Qiu (1997).

\section{INTRODUCTION}

Euler's gamma function, defined for positive real numbers $x$ by

$$
\Gamma(x)=\int_{0}^{\infty} e^{-t} t^{x-1} d t,
$$

is one of the most important functions in Analysis and Mathematical Physics. The history and the development of this function are described in an intriguing paper by P. J. Davis [6].

In the past, several authors proved many remarkable inequalities for $\Gamma$ (we refer to [3] and the references given therein). It is the aim of this article to present new inequalities for the gamma function which refine and extend some results given by W. Gautschi, G. D. Anderson, S.-L. Qiu, and the author.

In 1974, W. Gautschi [7] published a proof for an interesting inequality of V. R. Rao Uppuluri, who conjectured that for all positive real numbers $x$ the harmonic mean of $\Gamma(x)$ and $\Gamma(1 / x)$ is greater than or equal to 1 ,

$$
\frac{2}{1 / \Gamma(x)+1 / \Gamma(1 / x)} \geq 1 \quad(x>0) .
$$

Recently, the author [2] established the following closely related result:

$$
\frac{2}{1 /(\Gamma(x))^{2}+1 /(\Gamma(1 / x))^{2}} \geq 1 \quad(x>0) .
$$

Received by the editors March 10, 1998.

1991 Mathematics Subject Classification. Primary 33B15; Secondary 26D07.

Key words and phrases. Gamma function, psi function, power mean, inequalities.

(C)1999 American Mathematical Society 
It states that the harmonic mean of $(\Gamma(x))^{2}$ and $(\Gamma(1 / x))^{2}$ is always greater than or equal to 1.

If we denote by $M_{t}(a, b)$ the power mean of order $t$ of the positive real numbers $a$ and $b$, that is,

$$
M_{t}(a, b)=\left(\frac{a^{t}+b^{t}}{2}\right)^{1 / t} \quad(t \neq 0), \quad M_{0}(a, b)=(a b)^{1 / 2},
$$

then (1.1) and (1.2) are equivalent to

$$
M_{-1}(\Gamma(x), \Gamma(1 / x)) \geq 1 \quad \text { and } \quad M_{-2}(\Gamma(x), \Gamma(1 / x)) \geq 1 \quad(x>0),
$$

respectively. Since the function $t \mapsto M_{t}(a, b)$ is increasing on $\mathbb{R}$ (see [5, p. 159]), we obtain

$$
M_{-1}(\Gamma(x), \Gamma(1 / x)) \geq M_{-2}(\Gamma(x), \Gamma(1 / x)) \geq 1 \quad(x>0),
$$

which in particular shows that (1.2) is stronger than (1.1).

In view of (1.3) it is natural to look for further refinements of the UppuluriGautschi inequality (1.1). More precisely, we ask for the smallest real number $r$ such that

$$
M_{r}(\Gamma(x), \Gamma(1 / x)) \geq 1
$$

holds for all $x>0$. In Section 3 we prove that in (1.4) the best possible parameter $r$ is given by $r=1 / C-\pi^{2} /\left(6 C^{2}\right)$. Here and in what follows, $C=0.57721 \ldots$ denotes Euler's constant.

In 1997, G. D. Anderson and S.-L. Qiu [4] presented the following upper and lower bounds for $\Gamma(x)$ :

$$
x^{(1-C) x-1}<\Gamma(x)<x^{x-1} \quad(x>1) .
$$

Actually, the authors proved more. They established that the function $F(x)=$ $\log (\Gamma(x+1)) /(x \log (x))$ is strictly increasing on $(1, \infty)$ with $\lim _{x \rightarrow 1} F(x)=1-C$ and $\lim _{x \rightarrow \infty} F(x)=1$, which leads to (1.5). In Section 4 we prove a companion of (1.5). We show that if $x \in(1, \infty)$, then

$$
x^{\alpha(x-1)-C}<\Gamma(x)<x^{\beta(x-1)-C}
$$

is valid with the best possible constants $\alpha=\left(\pi^{2} / 6-C\right) / 2$ and $\beta=1$. This improves the bounds given in (1.5). Moreover, we show that if $x \in(0,1)$, then (1.6) holds with the best possible constants $\alpha=1-C$ and $\beta=\left(\pi^{2} / 6-C\right) / 2$.

\section{TWO LEMmas}

In order to prove our main theorems we need monotonicity and convexity properties of two functions which are connected with the psi function $\psi=\Gamma^{\prime} / \Gamma$ and its derivative. The first lemma extends a result of Gautschi [8], who established that $x \mapsto x^{2} \psi^{\prime}(x)$ is strictly convex on $(0,1 / 2)$.

Lemma 1. The function $x \mapsto x^{2} \psi^{\prime}(x)$ is strictly convex on $(0, \infty)$.

Proof. Let $x>0$ and $p(x)=x^{2} \psi^{\prime}(x)$. Then we obtain

$$
p^{\prime}(x)=2 x \psi^{\prime}(x)+x^{2} \psi^{\prime \prime}(x)
$$

and

$$
x^{-2} p^{\prime \prime}(x)=2 x^{-2} \psi^{\prime}(x)+4 x^{-1} \psi^{\prime \prime}(x)+\psi^{\prime \prime \prime}(x) .
$$


Using the integral representations

$$
\begin{gathered}
x^{-1}=\int_{0}^{\infty} e^{-x t} d t, \quad x^{-2}=\int_{0}^{\infty} e^{-x t} t d t \\
\psi^{\prime}(x)=\int_{0}^{\infty} e^{-x t} t\left(1-e^{-t}\right)^{-1} d t, \quad \psi^{\prime \prime}(x)=-\int_{0}^{\infty} e^{-x t} t^{2}\left(1-e^{-t}\right)^{-1} d t \\
\psi^{\prime \prime \prime}(x)=\int_{0}^{\infty} e^{-x t} t^{3}\left(1-e^{-t}\right)^{-1} d t
\end{gathered}
$$

(see [1, p. 260]), and the convolution theorem for Laplace transforms we get

$$
x^{-2} p^{\prime \prime}(x)=\int_{0}^{\infty} e^{-x t} q(t) d t
$$

where

$$
q(t)=2 t \int_{0}^{t} s\left(1-e^{-s}\right)^{-1} d s-6 \int_{0}^{t} s^{2}\left(1-e^{-s}\right)^{-1} d s+t^{3}\left(1-e^{-t}\right)^{-1} .
$$

Differentiation yields

$$
q^{\prime}(t)=2 \int_{0}^{t} s\left(1-e^{-s}\right)^{-1} d s-t^{2}\left(1-e^{-t}\right)^{-1}-t^{3} e^{-t}\left(1-e^{-t}\right)^{-2}
$$

and

$$
q^{\prime \prime}(t)=t^{2} e^{-2 t}\left(1-e^{-t}\right)^{-3} \sum_{k=3}^{\infty}(k-2) \frac{t^{k}}{k !}>0 \quad(t>0) .
$$

Hence, we obtain

$$
q^{\prime}(t)>\lim _{s \rightarrow 0} q^{\prime}(s)=0 \quad \text { and } \quad q(t)>\lim _{s \rightarrow 0} q(s)=0 \quad(t>0),
$$

so that $(2.1)$ implies that $p^{\prime \prime}$ is positive on $(0, \infty)$.

In what follows, $x_{0}=1.46163 \ldots$ denotes the abscissa of the minimum of $\Gamma$.

Lemma 2. The function $x \mapsto x \psi^{\prime}(x) / \psi(x)$ is decreasing and concave on $\left(1 / x_{0}, x_{0}\right)$.

Proof. In [7] and [8] it is proved that $x \mapsto x \psi(x)$ is negative, increasing and convex on $\left(1 / x_{0}, x_{0}\right)$. This implies that $x \mapsto-(x \psi(x))^{-1}$ is positive, increasing and convex on $\left(1 / x_{0}, x_{0}\right)$. Since $x \mapsto x^{2} \psi^{\prime}(x)$ is also positive, increasing and convex on $\left(1 / x_{0}, x_{0}\right)$ (a proof for the monotonicity is given in $\left.[7]\right)$, we conclude that the product

$$
x \mapsto-(x \psi(x))^{-1} \cdot x^{2} \psi^{\prime}(x)=-x \psi^{\prime}(x) / \psi(x)
$$

is increasing and convex on $\left(1 / x_{0}, x_{0}\right)$ (see $[10$, p. 16]).

\section{A POWER MEAN INEQUALITY FOR $\Gamma$}

We are now in a position to prove the following refinement of (1.3).

Theorem 1. The inequality

$$
M_{r}(\Gamma(x), \Gamma(1 / x)) \geq 1
$$

holds for all positive real numbers $x$ if and only if

$$
r \geq 1 / C-\pi^{2} /\left(6 C^{2}\right)=-3.20464 \ldots .
$$


Proof. First, we assume that inequality (3.1) is valid for all $x>0$. Then we obtain

$$
f_{r}(x)=\log \left(M_{r}(\Gamma(x), \Gamma(1 / x))\right) \geq 0=f_{r}(1) \quad(x>0) .
$$

Since $f_{r}^{\prime}(1)=0$, we conclude that

$$
f_{r}^{\prime \prime}(1)=(r-1)\left(\Gamma^{\prime}(1)\right)^{2}+\Gamma^{\prime}(1)+\Gamma^{\prime \prime}(1) \geq 0 .
$$

Using $\Gamma^{\prime}(1)=-C$ and $\Gamma^{\prime \prime}(1)=\pi^{2} / 6+C^{2}$, we get $r \geq 1 / C-\pi^{2} /\left(6 C^{2}\right)$.

Since $t \mapsto M_{t}(\Gamma(x), \Gamma(1 / x))$ is increasing on $\mathbb{R}$, it suffices to establish (3.1) with $r=1 / C-\pi^{2} /\left(6 C^{2}\right)$. We shall prove that the function

$$
g_{r}(x)=2\left(M_{r}(\Gamma(x), \Gamma(1 / x))\right)^{r}
$$

satisfies

$$
g_{r}(x)<g_{r}(1)=2 \quad(0<x \neq 1) .
$$

We have $g_{r}(x)=g_{r}(1 / x)$, so that it is enough to show that $g_{r}$ is strictly decreasing on $(1, \infty)$. Differentiation yields

$$
g_{r}^{\prime}(x)=r\left[(\Gamma(x))^{r-1} \Gamma^{\prime}(x)-x^{-2}(\Gamma(1 / x))^{r-1} \Gamma^{\prime}(1 / x)\right] .
$$

If $x>x_{0}$, then $\Gamma^{\prime}(1 / x)<0<\Gamma^{\prime}(x)$, so that $(3.2)$ implies $g_{r}^{\prime}(x)<0$. In order to prove that $g_{r}^{\prime}$ is also negative on $\left(1, x_{0}\right)$, we define for $x \in\left(1, x_{0}\right)$ :

$$
\begin{aligned}
h_{r}(x)= & r[\log (\Gamma(x))-\log (\Gamma(1 / x))]+\log (-\psi(x)) \\
& -\log (-\psi(1 / x))+2 \log (x) .
\end{aligned}
$$

A simple calculation reveals that the inequalities $g_{r}^{\prime}(x)<0$ and $h_{r}(x)<0$ are equivalent.

Differentiation leads to

$$
x h_{r}^{\prime}(x)=2+r u(x)+v(x)+v(1 / x),
$$

where

$$
u(x)=x \psi(x)+\frac{1}{x} \psi\left(\frac{1}{x}\right) \quad \text { and } \quad v(x)=\frac{x \psi^{\prime}(x)}{\psi(x)} .
$$

Since $1 / x_{0}<1 / x<x<x_{0}$, Lemma 2 and Jensen's inequality imply

$$
v(x)+v(1 / x) \leq 2 v\left(\frac{1}{2}(x+1 / x)\right),
$$

so that (3.3) and (3.4) yield

$$
x h_{r}^{\prime}(x) \leq 2+r u(x)+2 v\left(\frac{1}{2}(x+1 / x)\right) .
$$

In [2] it is proved that $u$ is strictly increasing on $(1, \infty)$, and from Lemma 2 we conclude that $v$ is decreasing on $\left(1 / x_{0}, x_{0}\right)$. Since $1 / x_{0}<1<\frac{1}{2}(x+1 / x)<x<x_{0}$, we obtain from (3.5) that

$$
x h_{r}^{\prime}(x)<2+r u(1)+2 v(1) .
$$

Using $\psi(1)=-C$ and $\psi^{\prime}(1)=\pi^{2} / 6$, we conclude that the sum on the right-hand side of (3.6) is equal to 0 . This leads to

$$
h_{r}^{\prime}(x)<0 \quad \text { and } \quad h_{r}(x)<h_{r}(1)=0 \quad\left(1<x<x_{0}\right) .
$$

Hence, we have

$$
g_{r}^{\prime}(x)<0 \quad\left(1<x \neq x_{0}\right)
$$


which implies that $g_{r}$ is strictly decreasing on $(1, \infty)$. This completes the proof of Theorem 1.

Remark. The proof of Theorem 1 reveals that the sign of equality holds in (3.1) with $r \geq 1 / C-\pi^{2} /\left(6 C^{2}\right)$ if and only if $x=1$.

\section{BOUNDS FOR $\Gamma$}

Our next theorem extends and sharpens double-inequality (1.5).

Theorem 2. If $x \in(0,1)$, then

$$
x^{\alpha(x-1)-C}<\Gamma(x)<x^{\beta(x-1)-C}
$$

with the best possible constants

$$
\alpha=1-C=0.42278 \ldots \quad \text { and } \quad \beta=\frac{1}{2}\left(\frac{\pi^{2}}{6}-C\right)=0.53385 \ldots
$$

And, if $x \in(1, \infty)$, then (4.1) holds with the best possible constants

$$
\alpha=\frac{1}{2}\left(\frac{\pi^{2}}{6}-C\right) \quad \text { and } \quad \beta=1 .
$$

Proof. In order to prove the inequalities (4.1) with the constant factors $\alpha$ and $\beta$, given in (4.2) and (4.3), respectively, we have to show that

(i) $f(x)=\log (\Gamma(x))-[(1-C)(x-1)-C] \log (x)>0$ on $(0,1)$,

(ii) $g(x)=(x-1-C) \log (x)-\log (\Gamma(x))>0$ on $(1, \infty)$, and

(iii) $h(x)=\log (\Gamma(x))-[\delta(x-1)-C] \log (x)$, with $\delta=\frac{1}{2}\left(\frac{\pi^{2}}{6}-C\right)$, is negative on $(0,1)$ and positive on $(1, \infty)$.

Differentiation yields

$$
f^{\prime}(x)=\psi(x+1)-(1-C)(1+\log (x))
$$

and

$$
x^{2} f^{\prime \prime}(x)=x^{2} \psi^{\prime}(x)-1-(1-C) x=y(x), \quad \text { say. }
$$

Lemma 1 implies that $y$ is strictly convex on $(0, \infty)$. Since $\lim _{x \rightarrow 0} y(x)=0, y(1)=$ $\pi^{2} / 6-2+C>0$ and $\lim _{x \rightarrow 0} y^{\prime}(x)=C-1<0$, we conclude that there exists a real number $\bar{x} \in(0,1)$ such that $y$ is negative on $(0, \bar{x})$ and positive on $(\bar{x}, 1)$. From (4.4) we obtain that $f$ is strictly concave on $(0, \bar{x})$ and strictly convex on $(\bar{x}, 1)$. Since $\lim _{x \rightarrow 0} f(x)=f(1)=f^{\prime}(1)=0$, we conclude that $f$ is positive on $(0,1)$. This proves (i).

Next, we establish (ii). We have

$$
g^{\prime}(x)=1-\frac{1+C}{x}+\log (x)-\psi(x)
$$

and

Using the estimate

$$
g^{\prime \prime}(x)=\frac{1}{x}+\frac{1+C}{x^{2}}-\psi^{\prime}(x)
$$

$$
\psi^{\prime}(x)<\frac{1}{x}+\frac{1}{2 x^{2}}+\frac{1}{6 x^{3}} \quad(x>0)
$$

(see [9]), we obtain for $x>1$ :

$$
g^{\prime \prime}(x)>\frac{1}{x^{3}}\left[\left(\frac{1}{2}+C\right) x-\frac{1}{6}\right]>0 .
$$


This implies

$$
g^{\prime}(x)>g^{\prime}(1)=0 \quad \text { and } \quad g(x)>g(1)=0 \quad(x>1) .
$$

Now, we prove (iii). Differentiation leads to

$$
h^{\prime}(x)=\psi(x)-\delta \log (x)-\delta+\frac{\delta+C}{x}
$$

and

$$
x^{2} h^{\prime \prime}(x)=x^{2} \psi^{\prime}(x)-\delta x-\delta-C=z(x), \quad \text { say } .
$$

From Lemma 1 we conclude that $z$ is strictly convex on $(0, \infty)$. Since $\lim _{x \rightarrow 0} z(x)=$ $1-\delta-C<0$ and $z(1)=0$, the function $z$ is negative on $(0,1)$ and positive on $(1, \infty)$. Hence, $(4.5)$ implies that $h$ is strictly concave on $(0,1)$ and strictly convex on $(1, \infty)$. Since $h(1)=h^{\prime}(1)=0$, we conclude that $h$ is negative on $(0,1)$ and positive on $(1, \infty)$.

From (4.1) we obtain

$$
\alpha<w(x)<\beta \quad(0<x \neq 1),
$$

where

$$
w(x)=\frac{1}{x-1}\left(C+\frac{\log (\Gamma(x))}{\log (x)}\right)
$$

The limit relations

$$
\lim _{x \rightarrow 0} w(x)=1-C, \quad \lim _{x \rightarrow 1} w(x)=\frac{1}{2}\left(\frac{\pi^{2}}{6}-C\right), \quad \text { and } \quad \lim _{x \rightarrow \infty} w(x)=1
$$

imply that the constant factors $\alpha$ and $\beta$, given in (4.2) and (4.3), respectively, are both best possible. This completes the proof of Theorem 2 .

Remark. From Theorem 2 we conclude that the inequalities

$$
((1-C) x-1) \log (x)<\log (\Gamma(x))<(x-1-C) \log (x)
$$

hold for $x>0$, while in [3] it is proved that the inequalities

$$
\begin{aligned}
\left(x-\frac{1}{2}\right) & \log (x)-x+\frac{1}{2} \log (2 \pi)+\sum_{k=1}^{2 n} \frac{B_{2 k}}{2 k(2 k-1)} x^{1-2 k} \\
& <\log (\Gamma(x))<\left(x-\frac{1}{2}\right) \log (x)-x+\frac{1}{2} \log (2 \pi)+\sum_{k=1}^{2 n+1} \frac{B_{2 k}}{2 k(2 k-1)} x^{1-2 k}
\end{aligned}
$$

are valid for $x>0$. Here $n \geq 0$ is an integer and $B_{2}, B_{4}, B_{6}, \ldots$ are Bernoulli numbers. A simple calculation shows that for all sufficiently small $x$ the bounds given in (4.6) are better than those given in (4.7), whereas for all sufficiently large $x$ the opposite is true. 


\section{REFERENCES}

1. M.Abramowitz and I. A. Stegun, eds., Handbook of Mathematical Functions with Formulas, Graphs and Mathematical Tables, Dover, New York, 1965. MR 94b:00012

2. H. Alzer, A harmonic mean inequality for the gamma function, J. Comput. Appl. Math. 87 (1997), 195-198. CMP 98:06

3. H. Alzer, On some inequalities for the gamma and psi functions, Math. Comp. 66 (1997), 373-389. MR 97e:33004

4. G. D. Anderson and S.-L. Qiu, A monotoneity property of the gamma function, Proc. Amer. Math. Soc. 125 (1997), 3355-3362. MR 98h:33001

5. P. S. Bullen, D. S. Mitrinović, and P. M. Vasić, Means and Their Inequalities, Reidel, Dordrecht, 1988. MR 89d:26003

6. P. J. Davis, Leonhard Euler's integral: A historical profile of the gamma function, Amer. Math. Monthly 66 (1959), 849-869. MR 21:5540

7. W. Gautschi, A harmonic mean inequality for the gamma function SIAM J. Math. Anal. 5 (1974), 278-281. MR 50:2570

8. W. Gautschi, Some mean value inequalities for the gamma function, SIAM J. Math. Anal. 5 (1974), 282-292. MR 50:2571

9. L. Gordon, A stochastic approach to the gamma function, Amer. Math. Monthly 101 (1994), 858-865. MR 95k:33003

10. A. W. Roberts and D. E. Varberg, Convex Functions, Academic Press, New York, 1973. MR 56:1201

Morsbacher Str. 10, 51545 Waldbröl, Germany 